\title{
A Novel SoC Feedback Control of ESS for Frequency Regulation of Fractional Frequency Transmission System with Offshore Wind Power
}

\author{
Boyang Zhao, Xifan Wang \\ State Key Laboratory on Electrical \\ Insulation and Power Equipment \\ Xi'an Jiaotong University \\ Xi'an, China \\ Email: zhaoboyang@stu.xjtu.edu.cn
}

\author{
Pierre Haessig \\ IETR \\ CentraleSupélec \\ Rennes, France
}

\begin{abstract}
The impact of high penetration of Renewable Energy Source (RES) and the decoupled power-frequency relationship raise the concern for RES participating in the grid frequency regulation. Energy Storage System (ESS) has shown its potential in power system with RES. This paper proposes a new control method of ESS considering the ancillary service efficiency and economical aspects. The proposed state of charge (SoC) feedback control is based on net-zero concept of frequency regulation model. A feedback gain is introduced to satisfy the grid regulations and economical index. The proposed control method is investigated over fractional frequency wind power system, which uses low frequency transmission lines or grids to transmit wind power. Simulations are done in Python and MATLAB/Simulink. The simulation results verify the effectiveness of proposed coordinate control strategy.
\end{abstract}

Index Terms-Energy Storage, frequency regulation, fractional frequency transmission system.

\section{INTRODUCTION}

Renewable Energy Source (RES) has been vastly integrated into power system during the last decades due to its sustainable and pollution-free feature. Widely using of double fed induction generator (DFIG) and permanent magnet synchronous generator (PSMG) leads to the dramatic increasing in installed capacity of wind turbine generation (WTG) where the global installation reaches $486.8 \mathrm{GW}$ in 2016 [1].

Fractional Frequency Transmission System (FFTS) was first proposed in 1994 and its feasibility has been analyzed [2], [3]. It is a very promising transmission method for wind power generation. By using low transmission frequency $(50 / 3 \mathrm{~Hz})$, this novel method is able to increase the transmission capacity and reduce the fluctuation at the connection point of wind farm [4]. Relevant experiments have been published in [5] as the system is called Fractional Frequency Wind Power System (FFWPS). Only onshore back-to-back MMC or $\mathrm{M}^{3} \mathrm{C}$ converter is used thus there is potential to incorporate energy storage system (ESS) into the converter to accomplish frequency conversion and energy storage function.

This work was supported by the State Grid Corporation of China under Grant 52094016000C.
High penetration of RES has brought the potential stability issues of power system considering the randomness and fluctuation of RES [6]-[8]. Wind power generators are usually connected to the grid with power electronic converters to improve the power quality. However, these converters lead to the decoupled relationship between grid frequency and output power which decrease the reliability of power system [9]. In addition, wind turbines usually operate in Maximum Power Point Tracking (MPPT) mode, which results in the unavailability for wind turbine participating in frequency regulation. And for a regional grid where there is high penetration of RES, the equivalent system inertia is reduced which risks the frequency safety and stability with large frequency nadir and rate of change of frequency (ROCOF) [10], [11].

Several control strategies have been proposed to deal with issues mentioned above [12]. The intuitive method is to emulate the inertia, which uses kinetic energy of rotor. The inertial response of DFIG using filed-oriented control is performed and analyzed in [13]. However, it should be noted that there is a recovery duration for wind turbine restoring rotation speed, during which the WTG will produce less power than the nominal.

WTG can operate in deloading mode to save the available power reserve. The deloading control can be achieved by overspeeding the WTG [12] or additional pitch control [14]. No recovery process is needed since WTG no longer operates in MPPT, but both methods may introduce mechanical abrasion thus increase repair rate.

Introducing ESS into RESs for frequency regulation has promising feature as reducing WTG generation loss. Due to its high investment and maintenance cost, ESS is only able to reduce frequency excursion in a short period of time. Therefore, a wind farm level coordinate control is needed. [15] has proposed a state of charge (SoC) feedback control of wind/ESS hybrid power system for frequency regulation. However, this strategy is only valid in an isolated power system since conventional generators respond to frequency fluctuation when SoC of ESS is too high or too low. Sizing of 
ESS is also crucial as it impacts the performance of frequency regulation. A battery energy storage system (BESS) is also optimized for frequency control in [16], but this does not hold ture when power system is large.

A SoC feedback control of ESS for frequency regulation has been proposed in this paper. ESS control should both maintain the health of ESS and frequency regulation. The novelty of this paper lies in the net-zero concept of ESS control. It is based on the fact that power generation and consumption are always balanced in a certain time interval but cannot always match at each time instant, which is called net-zero correction here. In this sense, the ESS can produce less when the $\mathrm{SoC}$ is low but it can compensate the error when SoC is high to keep a net-zero error during a short period. Thus grid frequency is in fluctuation around its nominal value due to this correction and frequency regulation service is done. A feedback gain concerning the SoC of ESS then is introduced to realize the net-zero correction. Furthermore, a low-pass filter is used to decompose frequency signal fed to WTG and ESS for full utilization of frequency regulation potential of these units.

The outline of this paper is as follows: Section II presents short description of simulation system modeling; Section III proposes a coordinate SoC feedback control; Section IV gives the simulation results and performance of coordinate SoC feedback control and conclusions are presented in Section V.

\section{SySTEM MODELING}

\section{A. Wind Turbine Generator Description}

DFIG and PMSG are now the most applied WTGs in wind power generation due to its variable rotation speed feature. The power electronic converters allow fast output control of WTGs. There are manufactures that provide high-capacity WTGs among which GE has published its DFIG dynamic model in [17]. Since the time constant of converter is much larger than mechanical time constant, power converter has been simplified as a first-order inertial element. Therefore, only mechanical model of DFIG will be discussed.

Single-mass rotor model is introduced to present the DFIG mechanical dynamics.

$$
2 H \omega_{m} \frac{\omega_{m}}{d t}=P_{m}-P_{e}
$$

$P_{m}$ and $P_{e}$ represent the mechanical power extracted from wind turbine and electrical active power of DFIG respectively. $\omega_{m}$ is the rotor rotation speed and $H$ is inertial constant. Mechanical power $P_{m}$ yields to (2) and depends on air density $\rho$, WT blades swept area $A$, wind speed $v_{\text {wind }}$, pitch angle $\beta$ , and tip-speed ratio $\lambda$.

$$
P_{m}=\frac{1}{2} C_{p}(\lambda, \beta) \rho A\left(v_{w i n d}\right)^{3}
$$

The performance coefficient $C_{p}(\lambda, \beta)$ is fitted by a fourth order polynomial, which is

$$
C_{p}(\lambda, \beta)=\sum_{i=0}^{4} \sum_{j=0}^{4} \alpha_{i, j} \beta^{i} \lambda^{j}
$$

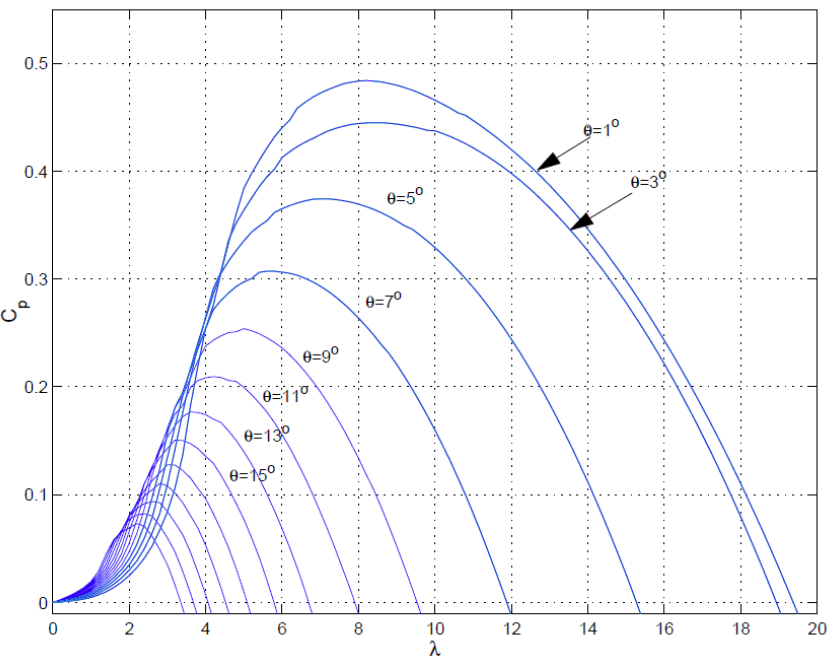

Fig. 1. Wind Power $C_{p}$ Curve

The coefficients and detailed control model of DFIG can be found in [17], [18]. Experimental $C_{p}(\lambda, \beta)$ curve is shown in Fig. 1.

\section{B. ESS Description}

Energy storage is used for frequency regulation in coordination with WTG. In this paper, the ESS is incorporated in the onshore back-to-back MMC or $\mathrm{M}^{3} \mathrm{C}$ converter of FFTS which can save construction cost of ESS. There are numerous types of utility scale battery or ultra-capacitor with fast transient can be introduced in converters mentioned above. Therefore, a system-level simplified battery ESS model is used where inverter connected to ESS is assumed to be lossless. ESS output $P_{E S S}$ is always satisfied when the request charing/discharging power $P_{r e q}$ can be reached and thus saturation is manged, as shown in (4) where $\Delta t$ is simulation time step. Note that when $P_{E S S}$ is positive (negative), ESS is discharging (charging).

$$
P_{E S S}= \begin{cases}\frac{S o C \times E_{E S S}}{\Delta t} & \text { for } P_{r e q}>\frac{S o C \times E_{E S S}}{\Delta t} \\ \frac{(S o C-1) \times E_{E S S}}{\Delta t} & \text { for } P_{r e q}<\frac{\left((S o C-1) \times E_{E S S}\right.}{\Delta t} \\ P_{r e q} & \text { for else }\end{cases}
$$

Practically, SoC of ESS is set in a certain interval to ensure lifetime, stability concerns and deterioration mitigation. Thus, typical SoC upper and lower limits are set to be 0.8 and 0.2 respectively.

$E_{E S S}$ here denotes the capacity of ESS. A 15 minutes of $2 \%$ wind farm installed capacity should be satisfied in wind farm participating frequency regulation as described in [19]. However, this capacity is the most conservative choice which means only ESS participates frequency regulation without WTGs in wind farm. Thus a smaller capacity ESS is used in this simulation which is 15 minutes of $1 \%$ wind farm installed capacity. 


\section{Load Description}

Imbalance in generation and consumption leads to frequency fluctuation. Therefore, the changing frequency can reflects some load changing information. A typical frequency pattern [20] is presented in Fig. 2.

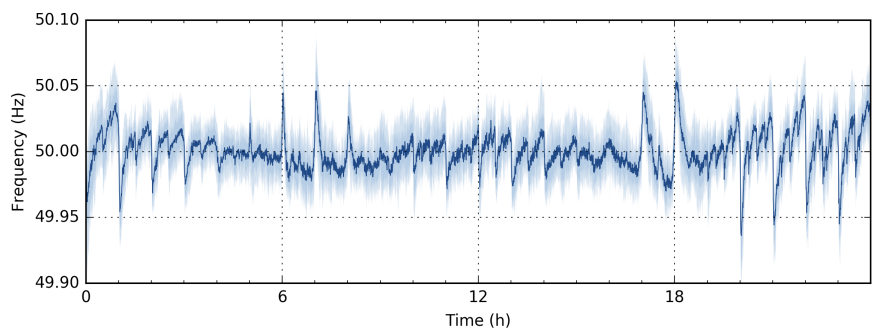

Fig. 2. Frequency Daily Pattern

Thus the power system load is defined in (5) where $k_{L}$ is static load-frequency coefficient and set to be 1.5. $f$ and $f_{N}$ are measured and nominal grid frequency respectively.

$$
\Delta P_{L}=-k_{L}\left(f-f_{N}\right)
$$

\section{Simulation System Description}

Classic Load-Frequency Control (LFC) is used in this paper. The structure of power system simulation model is depicted in Fig. 3. It consists of conventional steam turbine, DFIG wind farm, ESS, load and power system model. DFIG wind farm, ESS and load has already been described above. Steam turbine is composed by governor model and reheat turbine model. $R$ denotes the speed regulation parameter of the governor and $T_{G}$ is the mechanical governor response time. $K_{r}, T_{r}$ are steam turbine reheat constant and reheat time constant respectively. $T_{t}$ is the steam turbine time constant. Power system is represent by inertia constant $H$ and load-damping coefficient $D$. The parameters of power system and steam turbine are given in Appendix.

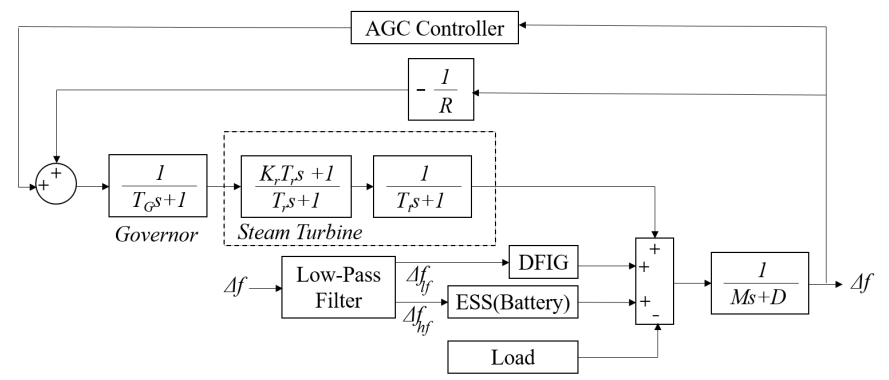

Fig. 3. Power system simulation model block diagram

Low-pass filter is introduced to decompose the frequency signal and high-frequency component $\Delta f_{h f}$ is fed to ESS for the fully utilization. Low-frequency component $\Delta f_{l f}$ is fed to DFIG for reduction in blade abrasion.

\section{COORdinAte CONTROL STRATEGY}

WTG should no longer operate in MPPT when it participates in frequency regulation service. Overspeed and pitch control are most discussed deloading control method for WTG participation frequency regulation [12], [21]. However, overspeed cannot correctly function without pitch control when wind speed is low so that coordinate control is necessary. As a result, this control method is implemented by DFIG deloading control realized by pitch control and ESS SoC feedback control.

\section{A. DFIG Deloading Control}

Pitch angle is considered to be smaller value in order to abstract more mechanical power from wind turbine. To deload wind turbine, an additional angle is introduced to maintain a certain deloading coefficient $k_{d e l}$. The required pitch angle value is approximately linear to desired deloading power [21]. A proportional gain is used to modify pitch angle $\theta$ respond to frequency deviation $\Delta f$ as emulation of droop control for steam turbine. The complete deloading control block diagram is shown in Fig. 4. $T_{\text {servo }}$ is the time constant of pitch blade actuator.

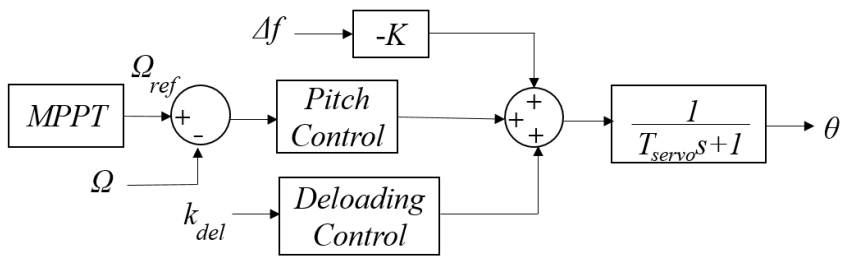

Fig. 4. Pitch Angle Control for frequency regulation

\section{B. SoC Feedback Control}

Capacity of battery ESS is limited thus its operating condition can be critical, where the upper and lower bounds are possibly reached. Practically, in order to expand the life of ESS, feedback control of ESS is introduced. As mentioned in Section II, the upper and lower limits are typically 0.8 and 0.2 respectively. The convention is always set the reference SoC to be 0.5 [15], however, it will lessen cycling life of ESS and impair the ESS performance since frequency deviation signal is independent of SoC.

In this paper, the adjustable reference $\mathrm{SoC}$ is implemented to fully utilize the battery ESS system. SoC reference value $S o C_{r e f}$ yields to (6), when SoC exceed its bounds, a penalty function in [22] is added to bring $\mathrm{SoC}$ into desired operation interval.

$$
S o C_{r e f}= \begin{cases}0.8 & \text { for } S o C>0.85 \\ 0.5 & \text { for } 0.15<S o C<0.85 \\ 0.2 & \text { for } S o C<0.15\end{cases}
$$

The complete coordinate control scheme is depicted in Fig. 5. DFIG wind farm responds to low-frequency component of frequency deviation and high-frequency component is fed to 
ESS where the total power of DFIG and ESS is the output of FFWPS. For the ESS control, An independent droop controller is introduced to simulate governor 5\% droop behavior. Also, it is noticed that there is a power error between desired and practical ESS output.

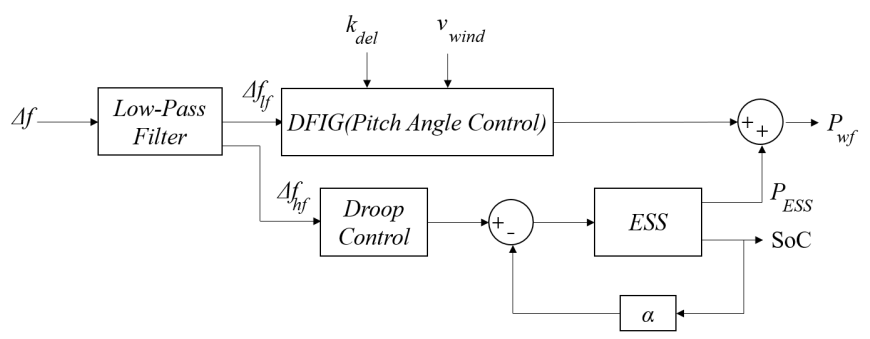

Fig. 5. Coordinate control strategy of ESS and FFWPS

In long-term operation, the root mean square value of this power error, also called PRMSE, should be minimized with feedback gain chosen carefully. In addition, load and generation may not be the same at each time instant. However, its must be equalized in a certain period of time. AGC is used to fulfill this function. Thus, a net-zero production and consumption is observed and net-zero of frequency deviation is achieved. Based on this concept, the optimization of PRMSE means the optimum control of wind farm participating frequency regulation.

\section{Simulation Results}

In this section, the proposed coordinate control scheme is verified in MATLAB/Simulink and Python, also the simulation results are presented. An FFWPS without ESS for participating frequency regulation is used as benchmark to outline the effectiveness of proposed control strategy.

\section{A. Original Simulation System}

A 594MW offshore wind farm is considered in this benchmark system. It is composed by 165 GE 3.6MW DFIGs as discussed above. 4 days have been randomly selected to represent the typical wind farm output during different seasons. The wind farm output curve is shown in Fig. 6. For the simplicity of simulation, only the spring wind output is considered in the following cases.

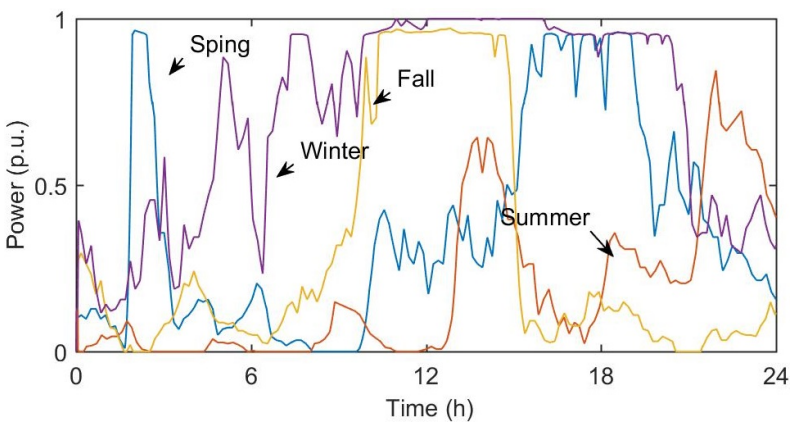

Fig. 6. Wind farm output power (p.u.)

\section{B. Benchmark System}

The power system is set to have wind power penetration as $20 \%$, deloading factor $k_{d e l}$ is $10 \%$ and the droop of wind farm is 5\%. Detailed parameters is given in Appendix. Load is described as Section II. Note that no ESS is implemented in this system, thus low-pass filter is no longer needed. Then the FFWPS output without ESS for frequency regulation is shown in Fig. 7 . Pitch angle is shown in Fig. 8 in comparison of MPPT mode.

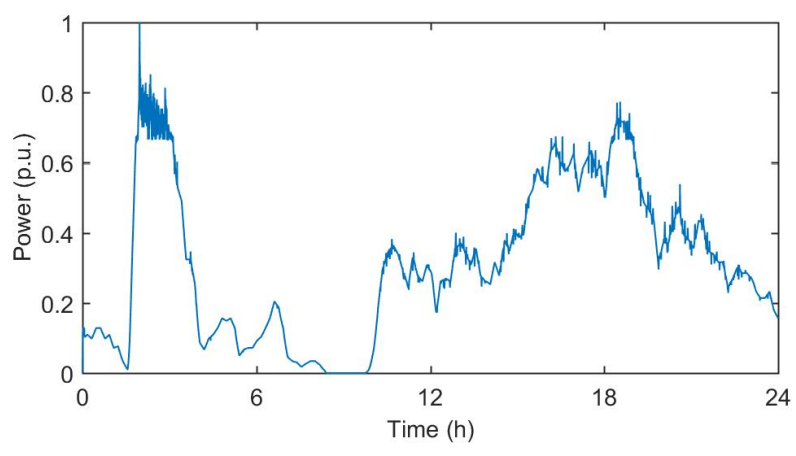

Fig. 7. FFWPS output without ESS for frequency regulation

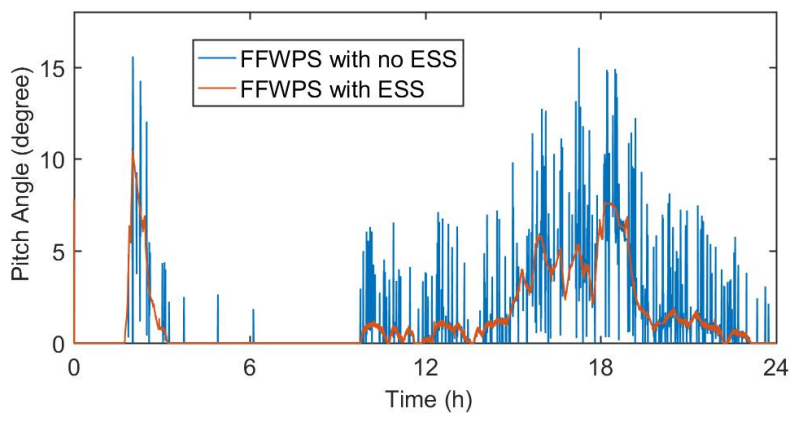

Fig. 8. DFIG pitch angle for frequency regulation

Obviously, by comparing Fig. 6 and Fig. 7,the power generation of wind farm is decreased due to deloading control. Fluctuation of wind farm is observed for the primary frequency regulation. A $10 \%$ deloading is necessary for frequency regulation. As described before, pitch control is applied for the deloading and frequency regulation so that the pitch angle is frequently adjusted as shown in Fig. 8. Mechanical abrasion may reduce the life of DFIG and introduce additional maintenance cost.

\section{Test System}

Incorporating ESS into FFWPS is beneficial to reducing mechanical abrasion and generation loss. Low-pass filter is implemented for the ESS application. Chosen of time constant $\tau$ of this filter needs to be optimized and is not discussed here. In this simulation, $\tau=15 \mathrm{~min}$, and other parameters remains same as benchmark system. Fig. 9 and Fig. 10 are the wind farm output power and pitch angle of DFIG. The ESS output reference in this case is shown in Fig. 11. 


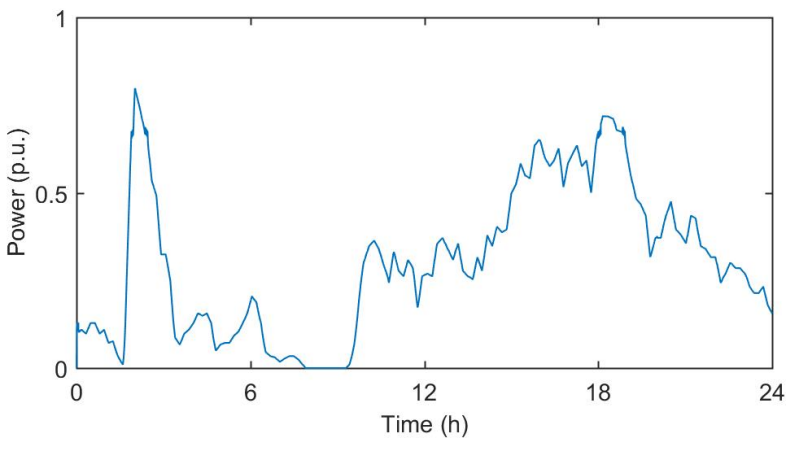

Fig. 9. FFWPS output with ESS for frequency regulation

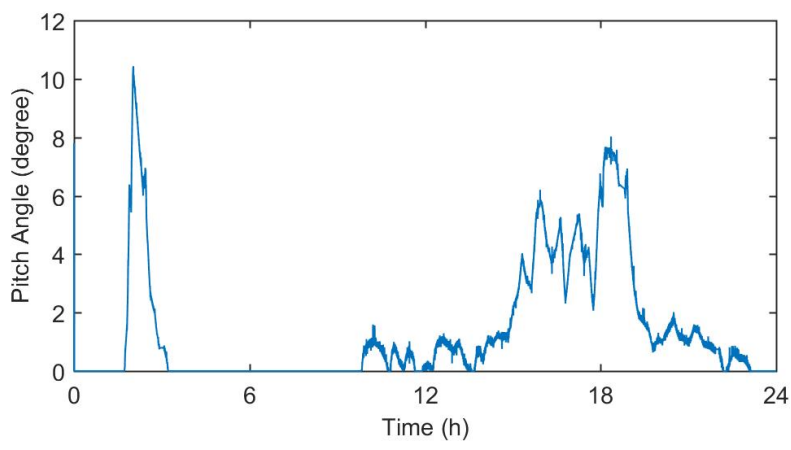

Fig. 10. DFIG pitch angle for frequency regulation

In this case, fluctuation of wind farm output is significantly reduced and pitch angle adjusting frequency is intensely decreased. This is due to the low-frequency component of frequency deviation is fed to DFIG. It is noted that deloading factor is not necessary to be $10 \%$ due to less reserve demand, which shows economical benefits with less generation loss.

ESS desire ouput power is responding to high-frequency deviation thus fast change is observed. Sizing of ESS can be simply decided as the maximum regulation power of ESS is satisfied. Note that this is the most conservative sizing criteria.

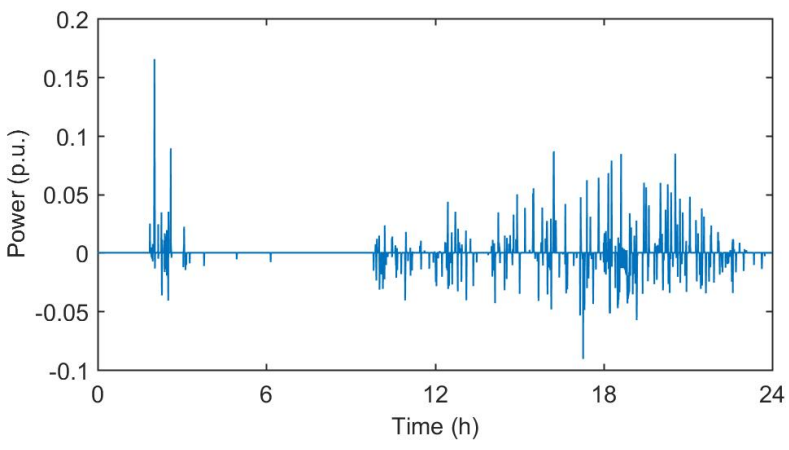

Fig. 11. ESS output power reference for frequency regulation

The SoC feedback control is implemented with feedback gain $\alpha$ chosen as 0.5. The simulation result is shown in Fig. 12. $P_{\text {droop }}$ is the reference power of ESS. A time interval from $12 \mathrm{~h}$ to $19 \mathrm{~h}$ is investigated. SoC of ESS is maintained in the desired bound and only small excursion is observed. The

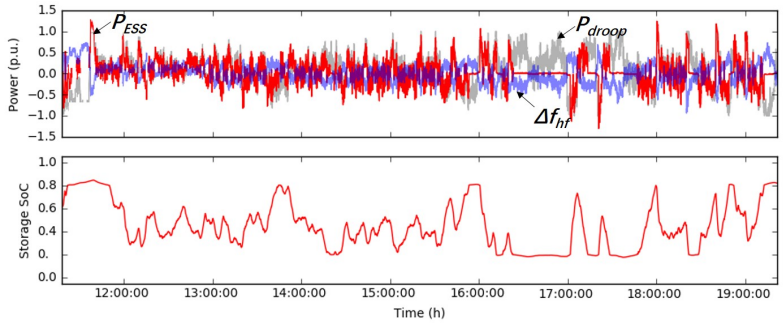

Fig. 12. ESS output of SoC feedback control with $\alpha=0.5$

ESS is not available from $16 \mathrm{~h} 30$ to $17 \mathrm{~h}$ as $P_{E S S}$ is nearly zero. This may introduce financial penalty in the sense of power market. However, the revenue of more generation with less deloading may compensate or even exceed this penalty. The system frequency is compared and shown in Fig. 13, the effectiveness is verified with small frequency deviation in SoC feedback control system.

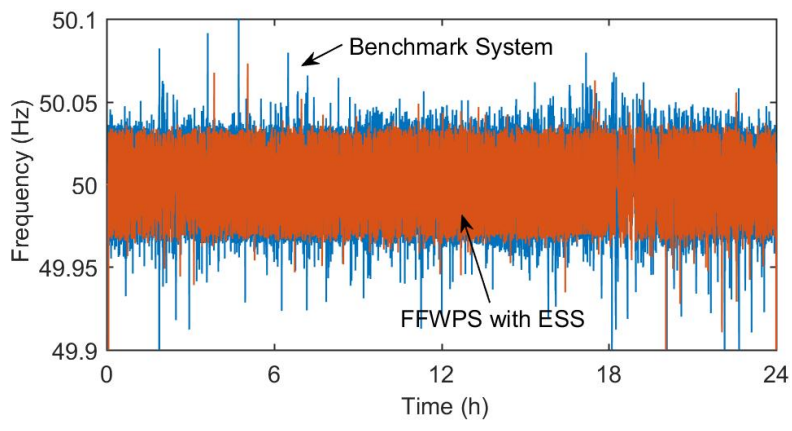

Fig. 13. System frequency

\section{CONClusion}

This paper has proposed a novel SoC feedback control of ESS for frequency regulation of FFTS with offshore wind power. Aggregate ESS is integrated in the onshore converter of back-to-back MMC or $\mathrm{M}^{3} \mathrm{C}$, which saves the construction cost of ESS. This strategy does not limited to the scale of power system since no feedback control signal is fed to conventional steam turbine. Low-pass filter is introduced to decompose frequency deviation so that DFIG and ESS are able to react to different frequency component. Coordinate control is implemented by simulation in MATLAB/Simulink and python also effectiveness is verified. Future research is needed for sizing of ESS and optimization of feedback gain. It is also a potential solution of wind farm/ESS hybrid control for frequency regulation when HVDC connection is used.

\section{APPENDIX}

a) Steam Turbine Parameters: Governor response time constant $T_{G}=0.2 \mathrm{~s}$, Steam turbine reheat constant $K_{r}=0.5 \mathrm{~s}$, Steam turbine reheat time constant $T_{r}=10.0 \mathrm{~s}$, Steam turbine time constant $T_{t}=0.3 \mathrm{~s}$, Governor speed regulation parameter $R=3.125$ 
b) Benchmark System Parameters: System inertia time constant $M=10.0$ s, Load-frequency coefficient $D=1$, AGC proportional gain $K_{p, A G C}=10.0 \mathrm{~s}$, AGC integration time constant $T_{i, A G C}=20.661 \mathrm{~s}$

\section{REFERENCES}

[1] GWEC, "Global wind report 2016," Global Wind Energy Council (GWEC), 2017. [Online]. Available: http://files.gwec.net/files/GWR2016.pdf?ref=Website

[2] X. Wang, "The fractional frequency transmission system," in Proceedings of International Sessions in IEE Japan, 1994, pp. 53-58.

[3] X. Wang and X. Wang, "Feasibility study of fractional frequency transmission system," IEEE Transactions on Power Systems, vol. 11, no. 2, pp. 962-967, May 1996

[4] S. Liu, X. Wang, L. Ning, B. Wang, M. Lu, and C. Shao, "Integrating offshore wind power via fractional frequency transmission system," IEEE Transactions on Power Delivery, vol. 32, no. 3, pp. 1253-1261, June 2017.

[5] X. Wang, X. Wei, and Y. Meng, "Experiment on grid-connection process of wind turbines in fractional frequency wind power system," IEEE Transactions on Energy Conversion, vol. 30, no. 1, pp. 22-31, March 2015.

[6] G. Lalor, A. Mullane, and M. O’Malley, "Frequency control and wind turbine technologies," IEEE Transactions on Power Systems, vol. 20 , no. 4, pp. 1905-1913, Nov 2005.

[7] R. Doherty, A. Mullane, G. Nolan, D. J. Burke, A. Bryson, and M. O'Malley, "An assessment of the impact of wind generation on system frequency control," IEEE Transactions on Power Systems, vol. 25, no. 1, pp. 452-460, Feb 2010.

[8] G. Delille, B. Francois, and G. Malarange, "Dynamic frequency control support by energy storage to reduce the impact of wind and solar generation on isolated power system's inertia," IEEE Transactions on Sustainable Energy, vol. 3, no. 4, pp. 931-939, Oct 2012.

[9] J. F. Conroy and R. Watson, "Frequency response capability of full converter wind turbine generators in comparison to conventional generation," IEEE Transactions on Power Systems, vol. 23, no. 2, pp. 649-656, May 2008.

[10] T. Kovaltchouk, V. Debusschere, S. Bacha, M. Fiacchini, and M. Alamir, "Assessment of the impact of frequency containment control and synthetic inertia on intermittent energies generators integration," in Proc. Eleventh Int. Conf. Ecological Vehicles and Renewable Energies (EVER), Apr. 2016, pp. 1-8.

[11] F. Wilches-Bernal, J. H. Chow, and J. J. Sanchez-Gasca, "A fundamental study of applying wind turbines for power system frequency control," IEEE Transactions on Power Systems, vol. 31, no. 2, pp. 1496-1505, March 2016.

[12] Y. z. Sun, Z. s. Zhang, G. j. Li, and J. Lin, "Review on frequency control of power systems with wind power penetration," in 2010 International Conference on Power System Technology, Oct 2010, pp. 1-8.

[13] A. Mullane and M. O'Malley, "The inertial response of inductionmachine-based wind turbines," IEEE Transactions on Power Systems, vol. 20, no. 3, pp. 1496-1503, Aug 2005.

[14] S. Ghosh, S. Kamalasadan, N. Senroy, and J. Enslin, "Doubly fed induction generator (dfig)-based wind farm control framework for primary frequency and inertial response application," IEEE Transactions on Power Systems, vol. 31, no. 3, pp. 1861-1871, May 2016.

[15] J. Dang, J. Seuss, L. Suneja, and R. G. Harley, "Soc feedback control for wind and ess hybrid power system frequency regulation," IEEE Journal of Emerging and Selected Topics in Power Electronics, vol. 2, no. 1, pp. 79-86, March 2014.

[16] P. Mercier, R. Cherkaoui, and A. Oudalov, "Optimizing a battery energy storage system for frequency control application in an isolated power system," IEEE Transactions on Power Systems, vol. 24, no. 3, pp. 1469 1477, Aug 2009.

[17] N. W. Miller, J. J. Sanchez-Gasca, W. W. Price, and R. W. Delmerico, "Dynamic modeling of ge 1.5 and $3.6 \mathrm{mw}$ wind turbine-generators for stability simulations," in 2003 IEEE Power Engineering Society General Meeting (IEEE Cat. No.03CH37491), vol. 3, July 2003, pp. 1977-1983 Vol. 3.

[18] N. R. Ullah, T. Thiringer, and D. Karlsson, "Temporary primary frequency control support by variable speed wind turbines-potential and applications," IEEE Transactions on Power Systems, vol. 23, no. 2, pp. 601-612, May 2008.
[19] E. N. On, "Germany grid code regulations for high and extra high voltage," Report ENENARHS2006, p. 46, 2006.

[20] RTE. (2017) Network frequency. [Online]. Available: https://clients.rtefrance.com/lang/an/clients_producteurs/vie/vie_frequence.jsp

[21] E. Loukarakis, I. Margaris, and P. Moutis, "Frequency control support and participation methods provided by wind generation," in Proc. IEEE Electrical Power Energy Conf. (EPEC), Oct. 2009, pp. 1-6.

[22] X. Li, D. Hui, and X. Lai, "Battery energy storage station (bess)based smoothing control of photovoltaic (pv) and wind power generation fluctuations," IEEE Transactions on Sustainable Energy, vol. 4, no. 2, pp. 464-473, April 2013. 